\title{
On the Sensitivity of Optimal Wave Energy Device Geometry to the Energy Maximizing Control System
}

\author{
Paula B. Garcia-Rosa, Member, IEEE, and John V. Ringwood, Senior Member, IEEE
}

\begin{abstract}
Wave energy converters (WECs) are traditionally designed independently of control systems. This usually involves matching the device frequency response to the predominant sea spectrum of a specific site. Energy maximizing control systems are subsequently employed to improve the energy conversion of the device for sea states other than the design sea state. It is well known that some control techniques have the ability to change the characteristics of a wave energy device, allowing it to improve the power absorption for frequencies other than the device natural frequency. However, while the controllers do their best to broaden the device frequency response, they can be limited in their capability to produce an overall optimal system. A different approach is studied in this paper, where iteration is performed between geometry optimization and control design. The aim is to investigate the implications of geometry optimization in the absence of control and the ultimate effect of incorporating the control design at the geometry optimization stage. Unconstrained control strategies are considered, namely, latching, declutching, and a model predictive control-like algorithm. It is shown that different optimal WEC geometries can be obtained depending on whether the device is controlled or not and the type of controller employed. The differences in overall power absorption characteristics of the optimized geometries are presented.
\end{abstract}

Index Terms-Control, optimal geometry, power optimization, wave energy.

\section{INTRODUCTION}

C URRENTLY, a wide variety of wave energy converters (WECs) have been proposed [1], [2] with little agreement on the best design (even for the same wave site) and little convergence in design principles. As is traditional in many control application areas, the fundamental application device (the WEC in this case) is designed by hydrodynamicists and/or wave energy device developers and then given to the control engineers to provide energy maximizing control. However, as with many control application areas, the overall system performance is compromised by the adoption of this separation of activities. A more holistic approach is indicated in Fig. 1, where iteration is performed between geometry optimization and control design, so that an overall optimal system is achieved.

Manuscript received October 05, 2014; revised February 13, 2015; accepted April 06, 2015. Date of publication May 11, 2015; date of current version December 11, 2015. This work was supported by the Science Foundation Ireland under Grant 12/RC/2302 for the Marine Renewable Energy Ireland (MaREI) Centre. Paper no. TSTE-00544-2014.

The authors are with the Centre for Ocean Energy Research, Maynooth University, Maynooth, Ireland (e-mail: paula.garciarosa@eeng.nuim.ie; john. ringwood@eeng.nuim.ie).

Color versions of one or more of the figures in this paper are available online at http://ieeexplore.iee.org.

Digital Object Identifier 10.1109/TSTE.2015.2423551
In general, the WEC geometry is designed for the prevailing sea conditions in a particular location, with control subsequently used to improve the energy conversion performance of the device for sea states other than the design sea state. This usually involves matching the device frequency response, or response amplitude operator (RAO) to the predominant sea spectrum. However, particular control strategies, subsequently employed and while doing their best to broaden the RAO in frequency range, can be limited in capability to produce an overall optimal system. Two cases in point highlight the issue. Latching control [3], [4], which delays the motion of a WEC by locking its position at the extreme (upper and lower) points of motion, has the ability to slow down the WEC in sea periods longer than the design period. On the other hand, declutching control [5] has the opposite effect-for intervals of the motion cycle, the WEC is "unloaded" allowing the response to be speeded up in sea periods shorter than the design period. Both latching and declutching produce composite device/control ("closed loop") systems, which are nonsymmetrical with respect to the predominant sea state.

Aiming to improve wave energy extraction, a number of studies have been done on device geometry optimization of WECs independently of the control system [6]-[10]. However, a preliminary study, examining the influence of latching control on the overall geometric optimization of a heaving cylinder, has been presented in [11].

This paper focuses on the implications of geometry optimization in the absence of control and the ultimate effect of the incorporation of control design at the device geometry optimization stage. A complete analysis for a WEC employing a variety of control strategies is presented. The strategies studied are: latching control, declutching control, and an optimal controller based on model-predictive control (MPC) [12]. The aim is to verify the different optimal WEC geometries corresponding to different control strategies, and the differences in overall power absorption characteristics of WEC geometries optimized with/without control considerations. The paper makes some significant recommendations based on the examples employed, which have important implications for how WECs are designed and optimized.

\section{Mathematical Modeling}

\section{A. Equations of Motion}

Here, we consider a generic point absorber with a generic power take-off (PTO) system, as is illustrated by Fig. 2. The point absorber is represented as a truncated vertical cylinder 


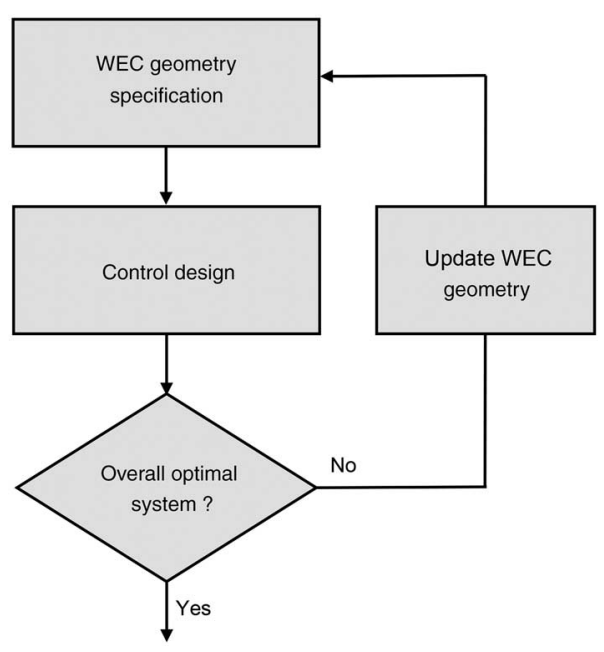

Fig. 1. Device optimization informed by the control system employed.

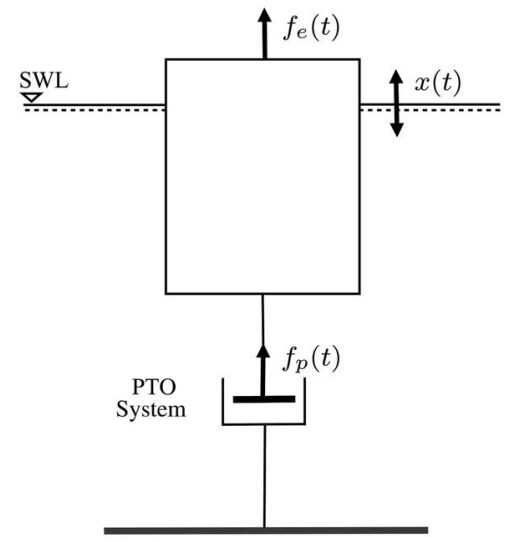

Fig. 2. Schematic of the generic heaving floating body.

with radius $r$ and draught $d$. In addition, linear hydrodynamic theory and heave oscillatory motion are assumed. In such a case, the motion of the floating body can be described by the Cummins' equation [13]

$$
\left[M+M_{r}(\infty)\right] \ddot{x}(t)+\int_{0}^{\infty} K(t-\tau) \dot{x}(\tau) d \tau+S_{h} x(t)=f(t)
$$

where $x(t)$ is the vertical position of the body, $M$ is the body mass, $M_{r}(\infty)$ is the infinite-frequency added mass coefficient, defined with the asymptotic values of the added masses at infinite frequency, $S_{h}$ is the hydrodynamic stiffness coefficient, and $f(t)$ is the total external force acting on the body. The kernel of the convolution term $K(t-\tau)$ is known as the fluid memory term and expressed as [13]

$$
K(t-\tau)=\frac{2}{\pi} \int_{0}^{\infty} B_{r}(\omega) \cos [\omega(t-\tau)] d \omega
$$

where $B_{r}(\omega)$ is the radiation damping coefficient and $\omega$ is the wave frequency. Assuming the body is freely floating in equilibrium, the body mass is equal to the mass of the displaced water in free flotation, i.e.,

$$
M=\rho \pi r^{2} d
$$

where $\rho$ is the water density. The external forces acting on the body

$$
f(t)=f_{e}(t)+f_{p}(t)
$$

are the wave excitation force $f_{e}(t)$ and the PTO force $f_{p}(t)$. The excitation force is calculated as $f_{e}(t)=\mathcal{F}^{-1}\left\{F_{e}(\omega) \eta(\omega)\right\}$, where $\eta(\omega)$ is the Fourier transform of the wave elevation and $F_{e}(\omega)$ is the excitation force transfer function.

If we consider a generic PTO system with a linear damper (coefficent $B_{p} \in \mathrm{R}_{+}$), then the PTO force is given by

$$
f_{p}(t)=-B_{p} \dot{x}(t)
$$

where $\dot{x}(t)$ is the vertical velocity of the body.

\section{B. Absorption of Wave Power}

In order to absorb energy from the waves, a point absorber must be equipped with a PTO system, as depicted in Fig. 2. Thus, the extracted energy and the mean extracted power by the WEC over a time range $T_{0}$ are, respectively, calculated as

$$
\begin{aligned}
J & =-\int_{0}^{T_{0}} \dot{x}(t) f_{p}(t) d t \\
P_{a} & =\frac{J}{T_{0}}
\end{aligned}
$$

where $f_{p}$ is the force applied by the PTO system.

The natural resonant period of the device is defined as

$$
T_{r}=2 \pi \sqrt{\frac{M+M_{r}(\infty)}{S_{h}}} .
$$

It is well known that there are two conditions for maximizing the wave energy conversion in monochromatic waves: 1) the phase condition and 2) the amplitude condition [14]. The phase condition is satisfied when the velocity of the body is in phase with the wave excitation force, which naturally occurs when the natural resonant period of the device $\left(T_{r}\right)$ is equal to the wave period. The amplitude condition indicates that there is an optimal value for the damping factor $B_{p}$ in (5). Assuming the phase condition is satisfied, then the optimal damping factor is equal to the radiation damping coefficient $B_{r}$ at the incident wave frequency [14].

Polychromatic waves (an approximation of real waves) are not defined by a single frequency in the time domain. Furthermore, the wave power varies in several time scales: from wave-to-wave (seconds), according to sea states (hours), and according to seasonal variations (months). Thus, the device geometry is usually designed for the prevailing sea conditions in a particular location, and a frequency that best characterizes the wave spectrum is selected. In this case, the natural resonant period $T_{r}$ and the damping factor $B_{p}$ can be tuned to the peak period $\left(T_{p}\right)$ or the energy period of the spectrum $\left(T_{e}\right)$. Control systems are usually subsequently employed to improve the energy conversion performance of the device for sea states other than the design sea state. 


\section{Optimization OF THE WEC GeOMETRY}

\section{A. Optimization Problem}

Here, our aim is to verify the different optimal geometries of a heaving cylinder that can be obtained when the geometry optimization process is done without any control strategy been employed to the WEC, and when different control strategies are employed to it. From (8), the parameters that define the natural period of the device are the mass $M$, the infinite added mass coefficient $M_{r}(\infty)$, and the hydrodynamic stiffness coefficient $S_{h}$. Such parameters are modified according to the radius $r$ and draught $d$ of the heaving cylinder.

Thus, the optimization of the device (Fig. 2) can be defined as: Determine the natural resonant period $T_{r}$, or equivalently, the radius $r$ and draught $d$ that maximize the average absorbed power $P_{a}$ (7) by the WEC with/without control strategies. Three different control strategies are considered in the analysis: 1) latching control; 2) declutching control; and 3) an optimal (MPC-like) controller.

\section{B. Latching Control}

The principle of latching control is to lock the motion of the body at the moment its velocity vanishes, and to wait for a favorable situation to release the body again. As a result, the body velocity is forced to be in phase with the wave excitation force. The determination of the time that the body is locked (latching duration $T_{L}$ ) is the problem to be solved.

Latching control "slows down" the natural response of the device when the wave period is longer than the device period, in order to force the velocity of the body and the excitation force to reach their extrema (maxima or minima) at the same time.

The latching time can be determined semianalytically for regular waves [15], [16]. However, for polychromatic waves, the concept of phase between excitation force and velocity is not well defined, in which case the optimization of the latching duration does not return a unique solution [15]. In this case, the latching duration (or equivalently, the unlatching time) can be optimized to synchronize the peak of the velocity with the peak of the excitation force [17] or to maximize the absorbed power [4]. In addition, a number of different strategies have been proposed to determine the best unlatching time for polychromatic waves, see e.g., [18]-[23].

In this study, we adopt the strategy proposed by Falcão [21] and later referred as threshold unlatching [22]. It consists of unlatching the body at the instant when the excitation force (or other reference variable) passes a chosen threshold [22]. Here, the threshold for the wave excitation force is set to zero.

\section{Declutching Control}

Declutching control, also called freewheeling or unlatching, was considered originally in [24]. It consists of unloading the device during some parts of the power cycle, which means that the PTO force is set equal to zero during such instants.

Under declutching, the PTO is disconnected (declutched) for a period following a maximum or minimum of position (i.e., velocity going to zero). Once the device has gained momentum under this no-load condition, the PTO is again connected, loading the system. The control problem, in this case, is the determination of the declutching duration for which the PTO is disconnected. In [5], an optimal command theory based on Pontryagin principle was proposed to determine the instants for clutching/declutching a WEC. Two different strategies are used in [25] to determine the instants for declutching: in the first one, the body is declutched approximately $T_{r} / 4$ before the next (predicted) peak of the excitation force, and in the second strategy, it is declutched after the excitation force passes a chosen threshold.

Declutching has also been studied in [26], which shows that declutching is an ideal strategy when the natural resonant period of the device is longer than the sea period. Effectively, loading and unloading the device allows it to "catch up" to the excitation force, which brings the device velocity into phase with the excitation force.

Following [26], we adopt the declutching control for the cases when the natural resonant period of the device is longer than the sea period. Here, the instants for clutching the device are determined by the instants the excitation force reaches its extremum. In this case, the PTO system is disengaged (declutched) when the device velocity vanishes, and it is engaged (clutched) again when the excitation force achieves its maximum or minimum value.

A combination of latching and declutching control is presented in [27] and a larger increase in the energy absorption is achieved, when compared to latching or declutching implemented independently. It was also shown that declutching works better with large values of the PTO damping, whereas latching works better with small values [27].

Latching and declutching control strategies are known as suboptimal control strategies, since they deal only with the phase condition for improving the wave energy absorption. Further improvements can be achieved if the PTO damping coefficient $B_{p}$ is adjusted according to the sea state, as has been done, e.g., in [26] and [28]. Both strategies implement an ON/OFF PTO force, usually through a braking mechanism (latching) or PTO bypass (declutching). In such cases, the PTO force is defined by (5) for the instants the PTO is ON. A different approach based on MPC is presented next. In this case, the PTO force is not defined by (5), it is obtained by solving an optimal energy absorption problem.

\section{MPC-Like Control Algorithm}

The control problem for the MPC-like control algorithm is defined as follows: to find the optimal profile for the PTO force which maximizes the total energy absorbed (6) over a time interval $T_{0}$, subjected to the equation of motion (1).

For this purpose, the control problem is discretized by approximating the velocity and the PTO force with a linear combination of basis functions. Such a combination results in a finite dimension optimization problem. Following the procedure from [12], trigonometric functions are chosen as basis functions. Then, the PTO force and the velocity 
are, respectively, approximated with the truncated zero-mean Fourier series

$$
\begin{aligned}
\dot{x}(t) & \approx \sum_{n=1}^{N / 2} \nu_{n}^{c} \cos \left(n \omega_{0} t\right)+\nu_{n}^{s} \sin \left(n \omega_{0} t\right) \\
f_{p}(t) & \approx \sum_{n=1}^{N / 2} \phi_{n}^{c} \cos \left(n \omega_{0} t\right)+\phi_{n}^{s} \sin \left(n \omega_{0} t\right)
\end{aligned}
$$

where $\omega_{0}$ is the fundamental frequency of the Fourier series and $N$ is the total number of components.

The best approximation of the solution for the equation of motion (1) is sought by applying the Galerkin method (see [29] for more details), and the result is the linear system

$$
G X=P+E
$$

where $X, P, E$ are, respectively, the vectors of the Fourier coefficients of the velocity, PTO force, and excitation force, and $G$ is the matrix of hydrodynamical coefficients of the system. The set of vectors is arranged as

$$
\begin{aligned}
X & =\left[\nu_{1}^{c}, \nu_{1}^{s}, \nu_{2}^{c}, \nu_{2}^{s}, \ldots, \nu_{\frac{N}{2}}^{c}, \nu_{\frac{N}{2}}^{s}\right]^{T} \\
P & =\left[\phi_{1}^{c}, \phi_{1}^{s}, \phi_{2}^{c}, \phi_{2}^{s}, \ldots, \phi_{\frac{N}{2}}^{c}, \phi_{\frac{N}{2}}^{s}\right]^{T} \\
E & =\left[\epsilon_{1}^{c}, \epsilon_{1}^{s}, \epsilon_{2}^{c}, \epsilon_{2}^{s}, \ldots, \epsilon_{\frac{N}{2}}^{c}, \epsilon_{\frac{N}{2}}^{s}\right]^{T} .
\end{aligned}
$$

The matrix $G \in \mathrm{R}^{N \times N}$ is defined as

$$
G=\left[\begin{array}{cccccc}
D_{1} & C_{1} & 0 & \cdots & 0 & 0 \\
-C_{1} & D_{1} & 0 & \cdots & 0 & 0 \\
0 & 0 & \ddots & & \vdots & \vdots \\
\vdots & \vdots & & \ddots & 0 & 0 \\
0 & 0 & 0 & 0 & D_{N / 2} & C_{N / 2} \\
0 & 0 & 0 & 0 & -C_{N / 2} & D_{N / 2}
\end{array}\right]
$$

with

$$
\begin{aligned}
D_{n} & =B_{r, n}\left(n \omega_{0}\right) \\
\text { and } C_{n} & =n \omega_{0}\left(M+M_{r, n}\left(n \omega_{0}\right)\right)-\mathrm{S}_{h, n} /\left(n \omega_{0}\right) .
\end{aligned}
$$

Assuming the wave excitation force is known completely into the future and $G$ is nonsingular, the vector of the optimal PTO force $P^{*}$ is obtained by solving the following optimization problem [30]

$$
P^{*}=\arg \max _{P}\left(-P^{T} G^{-1} P-P^{T} G^{-1} E\right) .
$$

\section{RESULTS}

\section{A. Simulation Parameters}

Simulation results with polychromatic waves are presented in this section. The polychromatic waves are calculated as a linear combination of monochromatic waves using a Bretschneider spectral envelope, defined by the significant wave height $H_{s}$, peak period $T_{p}$, and random phases.
The optimal geometries for each of the cases: 1) uncontrolled device (UD); 2) latching-controlled device (LD); declutchingcontrolled device (DD); and MPC-controlled device (MPCD) are determined via simulations with a complete set of possible values of radius $r$ and draught $d$, in which 200 different cylinders are considered. As a result, a clear overview of the mapping between the absorbed power $P_{a}$ and the optimization parameters can be observed. The ranges of both the radii and draughts go from 2 to $20 \mathrm{~m}$, in steps of $1 \mathrm{~m}$ (radius) and $2 \mathrm{~m}$ (draught).

The coefficients $F_{e}(\omega), B_{r}(\omega), M_{r}(\infty)$, and $S_{h}$ for all the cylinders are computed using the boundary element solver WAMIT [31]. The setup parameters for WAMIT are $\rho=$ $1025 \mathrm{~kg} / \mathrm{m}^{3}$, water depth: $60 \mathrm{~m}$, gravity: $9.81 \mathrm{~m} / \mathrm{s}^{3}$, and frequency range: $(\pi / 128: \pi / 128: \pi) \mathrm{rad} / \mathrm{s}$. For the MPCD, the simulation parameters are $N=128$ and $\omega_{0}=0.0304 \mathrm{rad} / \mathrm{s}$. For all simulations $T_{0}$ is set to $1200 \mathrm{~s}$, which is the usual recording time used to obtain real statistical values of a wave field, and to characterize a sea state [32].

Different criteria are used to tune the PTO damping coefficient $B_{p}$ for the uncontrolled case and the latching/declutching strategies. For the uncontrolled case

$$
B_{p}=\sqrt{\left(B_{r}(\omega)\right)^{2}+\left(\omega\left(M+M_{r}(\omega)\right)-S_{h} / \omega\right)^{2}}
$$

which represents optimal linear damping when the body is subjected to incident regular waves [14]. Irregular waves are not defined by a single frequency in the time domain. For this reason, a frequency that best characterizes the wave spectrum should be selected. Here, $B_{p}$ is tuned to the peak frequency of the spectrum $\omega_{p}$ for each sea state.

For the latching and declutching control cases, we have determined the optimal damping for each one of the geometries and sea states. In these cases

$$
B_{p}=D_{m} / 100 M
$$

and the problem to be solved is to determine the coefficient $D_{m}$, which optimizes the absorbed power for a specific geometry, control strategy, and sea state. Then, the optimal $D_{m}$ coefficient determined for each one of the 200 geometries is applied at the main evaluation loop (the loop that evaluates the optimal geometry). To illustrate how the coefficients $D_{m}$ were calculated, Fig. 3 shows the evolution of the power absorbed versus $D_{m}$ for some specific geometries. In these cases, $H_{s}=1 \mathrm{~m}$ and $T_{p}=7 \mathrm{~s}$.

\section{B. Simulation Results}

Figs. 4 and 5 illustrate the evolution of the absorbed power $P_{a}$ for different values of $r$ and $d$ for each of the four cases (UD, LD, DD, and MPCD). The figures also show the level curves of $P_{a}$ under the surface plot, allowing easy identification of the optimal cylinder parameters. The devices are subject to polychromatic waves with $H_{s}=1 \mathrm{~m}$ and peak periods of 5 and $7 \mathrm{~s}$, respectively. Table I summarizes the optimal values $\left(r^{*}, d^{*}\right.$, $T_{r}^{*}$ ) and the absorbed power $P_{a}$ obtained for these cases.

The results show that the optimal geometries obtained with latching control are different from the optimal geometries 


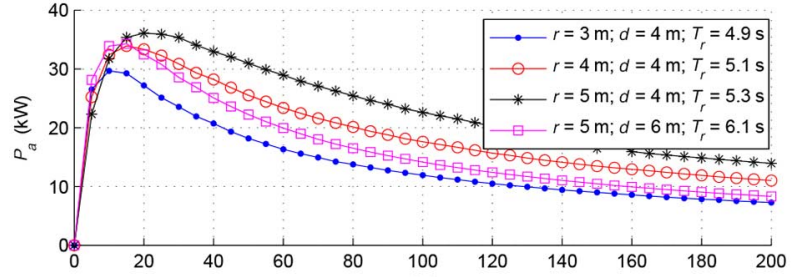

(a)

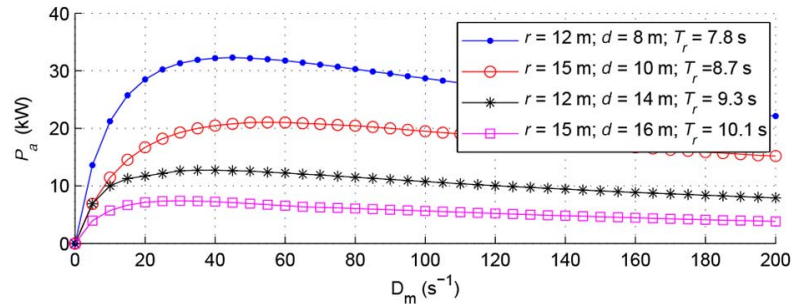

(b)

Fig. 3. Evolution of the power absorbed versus the coefficient $D_{m}$ : optimization of the optimal linear damping for the (a) LD and (b) DD.

obtained when no control is applied. As expected, the natural resonant period of the uncontrolled cases is very close to the peak period $T_{p}$ of the wave spectrum, whereas the optimal values of $T_{r}$ are slightly smaller for the LDs. In this case, the optimal values for $r$ and $d$ are also smaller than the uncontrolled case. It is worth mentioning that larger differences between LDs and UDs can be found, if smaller PTO damping coefficients are applied to the WEC, as showed in [11].

The optimal geometries obtained with declutching control are slightly different from the uncontrolled case. For the MPCD, the evolution of the absorbed power is completely different from all of the three other cases (UD, LD, and DD). The controller in this case, optimizes the absorbed power for all the geometries, regardless of the resonant period of the device and the wave period. In some respects, it can be considered that the MPC-like controller combines the best features of both latching and declutching, since it has significantly more degrees of freedom in parameterizing the control signal, compared to latching and declutching. The absorbed power for the best geometry is not significantly different from other geometries in the map [Figs. 4(d) and 5(d)].

In order to illustrate the effect of each of the control strategies in the device, Fig. 6 illustrates the motion of the device controlled by latching, declutching, and the MPC-like algorithm, respectively, for a chosen geometry. In these cases, $H_{s}=1 \mathrm{~m}$ and $T_{p}=7 \mathrm{~s}$. The MPC-like algorithm optimizes the PTO force applied to the device on a wave-to-wave time scale, resulting in more exaggerated motion of the body, and consequently, more absorbed power than the latching and declutching strategies. Conceptually, latching and declutching are suboptimal control methods aiming to achieve the optimum phase condition between the device velocity and the wave excitation force.

\section{Discussion}

Table II shows the relative size of the devices optimized for irregular waves with $H_{s}=1 \mathrm{~m}$ and $T_{p}=7 \mathrm{~s}$ and the various

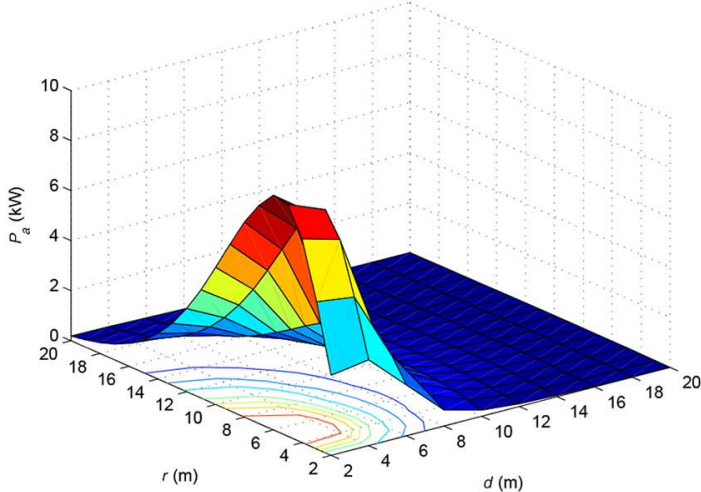

(a)

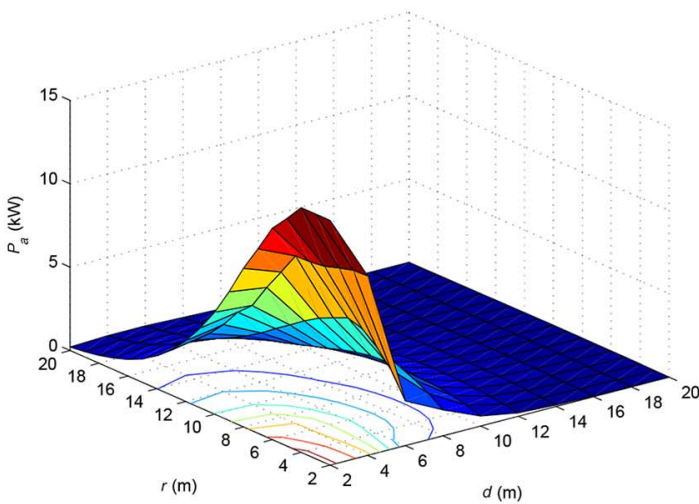

(b)

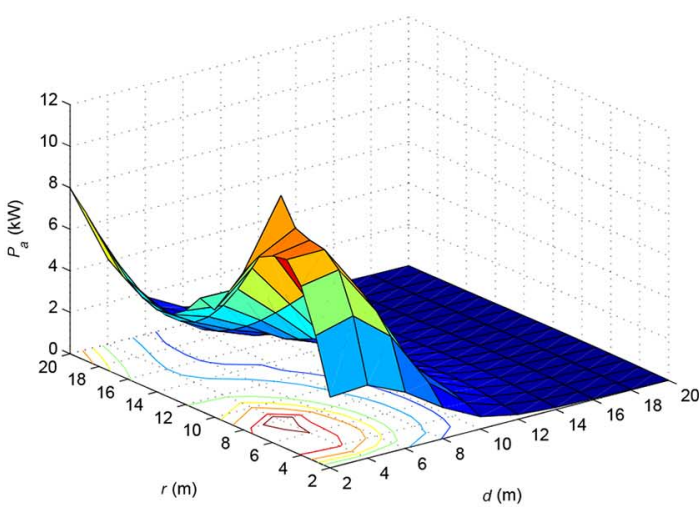

(c)

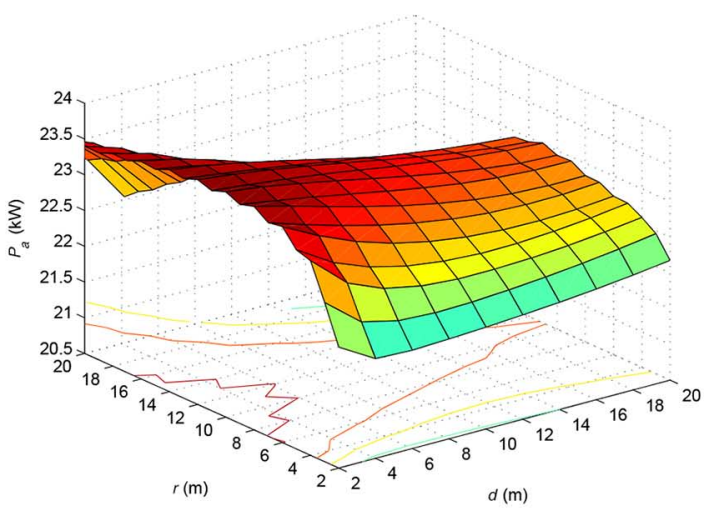

(d)

Fig. 4. Evolution of the absorbed power for different values of $r$ and $d$ (a) UD; (b) LD; (c) DD; and (d) MPCD (polychromatic waves: $H_{s}=1 \mathrm{~m} ; T_{p}=5 \mathrm{~s}$ ). 


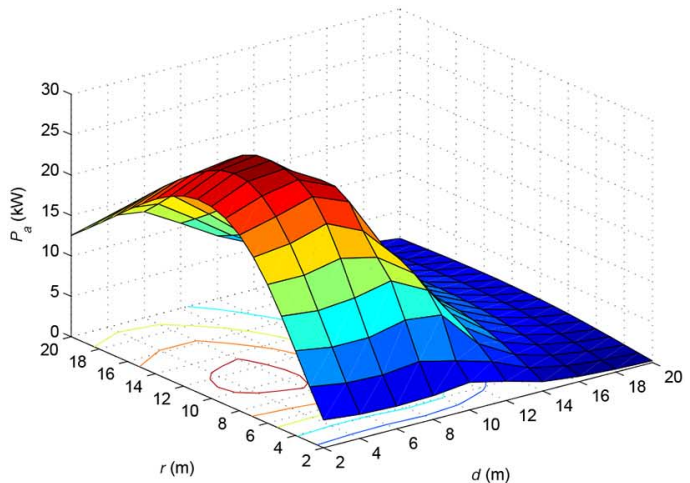

(a)

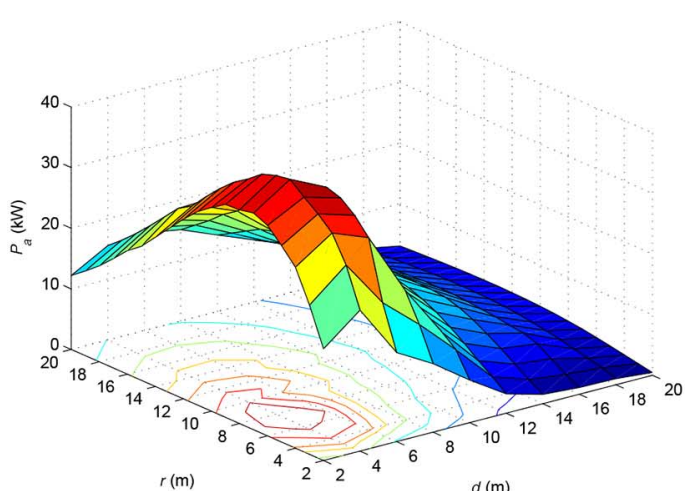

(b)

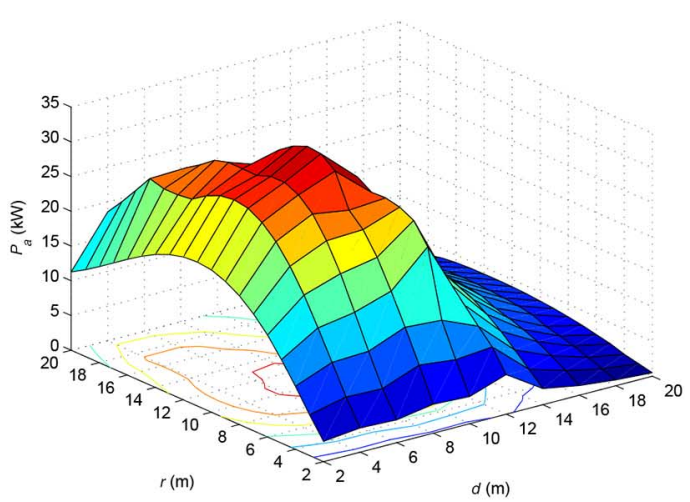

(c)

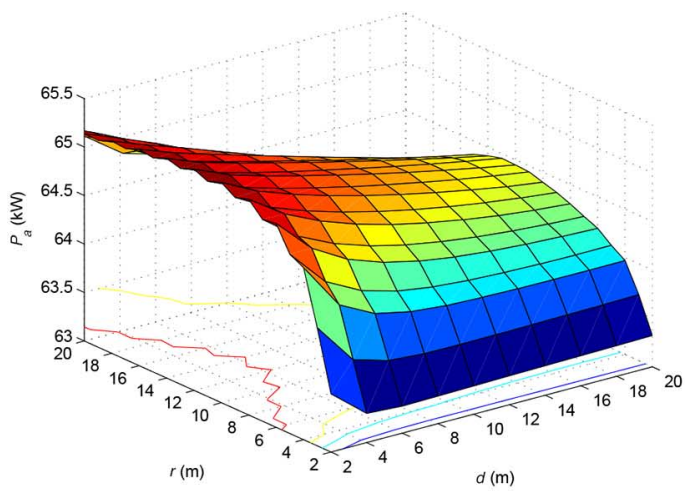

(d)

Fig. 5. Evolution of the absorbed power for different values of $r$ and $d$ (a) UD; (b) LD; (c) DD; (d) MPCD (polychromatic waves: $H_{s}=1 \mathrm{~m}$; $T_{p}=7 \mathrm{~s}$ ).
TABLE I

Optimal VAlues of $r, d$, AND $T_{r}$ OF THE CYLINDERS AND ABSORBED POWER $P_{a}$ FOR UD, LD, DD, AND MPCD (POLYCHROMATIC WAVES WITH $H_{s}=1 \mathrm{~m}$ )

\begin{tabular}{|c|c|c|c|c|}
\hline \multirow{2}{*}{} & \multicolumn{4}{|c|}{$T_{p}=5 \mathrm{~s}$} \\
\cline { 2 - 5 } & UD & LD & DD & MPCD \\
\hline$r^{*}(\mathrm{~m})$ & 6 & 2 & 8 & 10 \\
\hline$d^{*}(\mathrm{~m})$ & 4 & 2 & 4 & 4 \\
\hline$T_{r}^{*}(\mathrm{~s})$ & 5.55 & 3.68 & 5.91 & 6.24 \\
\hline$P_{a}(\mathrm{~kW})$ & 9.38 & 14.67 & 10.82 & 23.58 \\
\hline \hline & \multicolumn{5}{|c|}{$T_{p}=7 \mathrm{~s}$} \\
\cline { 2 - 5 } & UD & LD & DD & MPCD \\
\hline$r^{*}(\mathrm{~m})$ & 11 & 6 & 12 & 12 \\
\hline$d^{*}(\mathrm{~m})$ & 6 & 4 & 8 & 4 \\
\hline$T_{r}^{*}(\mathrm{~s})$ & 7.06 & 5.55 & 7.79 & 6.56 \\
\hline$P_{a}(\mathrm{~kW})$ & 26.34 & 39.73 & 33.29 & 65.13 \\
\hline
\end{tabular}

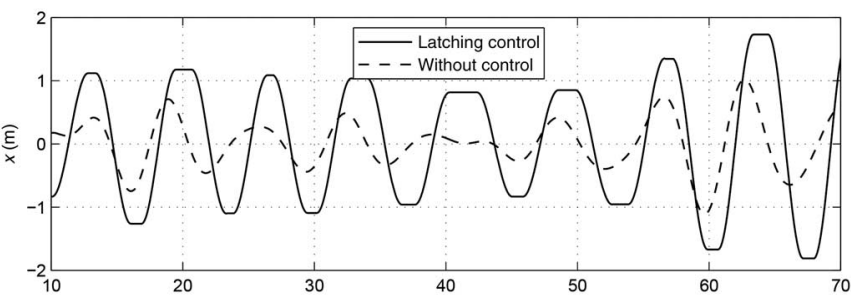

(a)

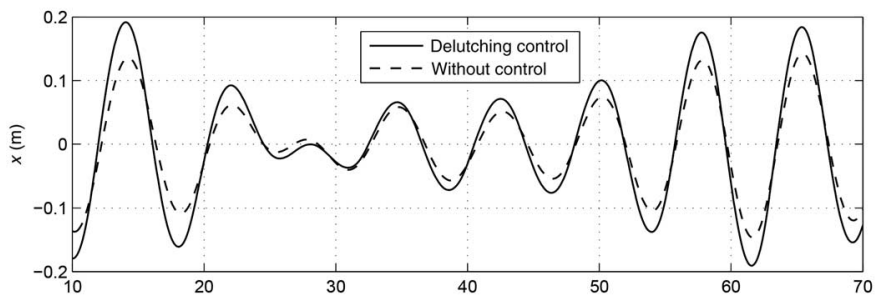

(b)

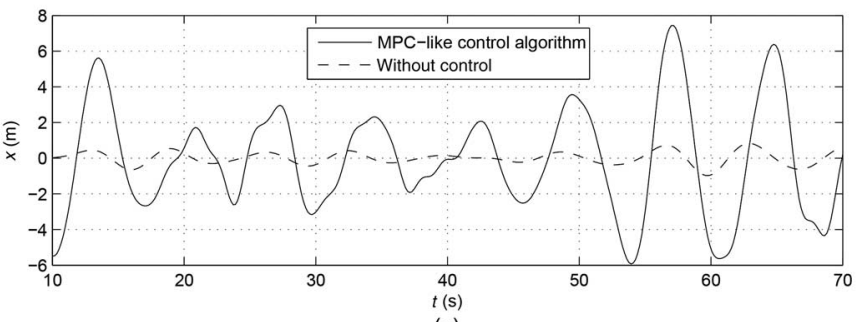

(c)

Fig. 6. Motion of a heaving cylinder with control (solid lines) and without control (dashed line) (a) $\mathrm{LD}\left(r=4 \mathrm{~m} ; d=4 \mathrm{~m} ; T_{r}=5.1 \mathrm{~s}\right)$; (b) DD $(r=12 \mathrm{~m}$; $\left.d=14 \mathrm{~m} ; T_{r}=9.3 \mathrm{~s}\right) ;$ and $(\mathrm{c}) \operatorname{MPCD}\left(r=4 \mathrm{~m} ; d=4 \mathrm{~m} ; T_{r}=5.1 \mathrm{~s}\right)$.

control scenarios. For example, it is interesting that the optimal latched device has a significantly larger power/volume ratio than the uncontrolled case. However, a reduced device size is not evident for all control cases and many other considerations need to be taken into account (such as stroke length, PTO force limits, etc.,) in any comparative economic assessment.

Table III shows the power absorbed for the UD, LD, DD, and MPCD for all the optimal geometries (GEO A, GEO B, GEO C, and GEO D, respectively). When we compare the power absorbed for the LD with GEO A to the power absorbed with GEO B, an improvement of $18 \%$ is obtained for GEO B. For the 
TABLE II

Relative Size of the Various Optimized Devices and Relative Power Captured Per Unit Volume

\begin{tabular}{|c|c|c|c|c|c|c|}
\hline Scenario & Geometry & $r(\mathrm{~m})$ & $d(\mathrm{~m})$ & $V_{o}\left(\mathrm{~m}^{3}\right)$ & $P_{a}(\mathrm{~W})$ & $P_{a} / V_{o}$ \\
\hline UD & GEO A & 11 & 6 & 2280.80 & 26340 & 11.55 \\
\hline LD & GEO B & 6 & 4 & 452.39 & 39730 & 87.82 \\
\hline DD & GEO C & 12 & 8 & 3619.11 & 33290 & 9.20 \\
\hline MPCD & GEO D & 12 & 4 & 1809.56 & 65130 & 35.99 \\
\hline
\end{tabular}

TABLE III

Power Absorbed $(\mathrm{kW})$ FOR VARIOUs CONTROL STRATEgIES With DifFERENT OPTIMAL DeVICE GeOMETRIES

\begin{tabular}{|c|c|c|c|c|}
\hline \multirow{2}{*}{ Geometry } & \multicolumn{4}{|c|}{ Scenario } \\
\cline { 2 - 5 } & UD & LD & DD & MPCD \\
\hline GEO A & 26.34 & 33.75 & 30.30 & 64.95 \\
\hline GEO B & 17.13 & 39.73 & 19.05 & 64.75 \\
\hline GEO C & 21.88 & 26.54 & 33.29 & 64.92 \\
\hline GEO D & 25.55 & 33.10 & 28.02 & 65.12 \\
\hline
\end{tabular}

DD case, when we compare the power absorbed with GEO A to the power with GEO C, an improvement of $10 \%$ is obtained for GEO C. Notice that different strategies based on optimal command theory to compute the instants for clucthing/declutching, e.g., [5], may be adopted to verify if higher improvements can be obtained for declutching control.

It can also be noted that the optimal geometry obtained for the uncontrolled case (GEO A), e.g., is not optimal if one wants, afterward, to apply latching control on it. The converse remark can also be stated for the optimal geometry obtained with the LD (GEO B), the natural mechanical properties of the optimal shape are no longer optimal for the considered sea-state, if one does not want, afterward, to apply latching control to the device.

Nevertheless, the MPC-like algorithm optimizes the wave energy absorption regardless of the geometry of the device. Four different geometries result in about the same amount of power. Such behavior is in accordance with one of the wave energy principles from Falnes [14], which states that a big body and a small body may produce equally large waves (and hence convert wave energy into useful energy), provided the smaller body oscillates with larger amplitude. But note that no constraints are considered here. For practical application studies, constraints on the amplitude of the device motion or on the PTO force should be taken into account.

Fig. 7 compares the RAOs of the optimal geometries. Latching control broadens the bandwidth of the RAO for wave periods longer than the device resonant period. Since GEO B is smaller than GEO A, the benefit of applying such strategy is now also extended for periods between 5 and 7 s. If GEO A has been considered, the benefit of latching control would be seen only for periods longer than $7 \mathrm{~s}$. The bandwidth for the declutching control is slightly broader than the UD case for wave periods shorter than the device resonant period [Fig. 7(a)]. However, the benefit of the declutching control is seen mostly around the resonant period of the geometry, as it has also been shown in [27]. On the other hand, the MPCD broadens the bandwidth of the RAO regardless of the period and geometry of the device, as is illustrated in Fig. 7(b).

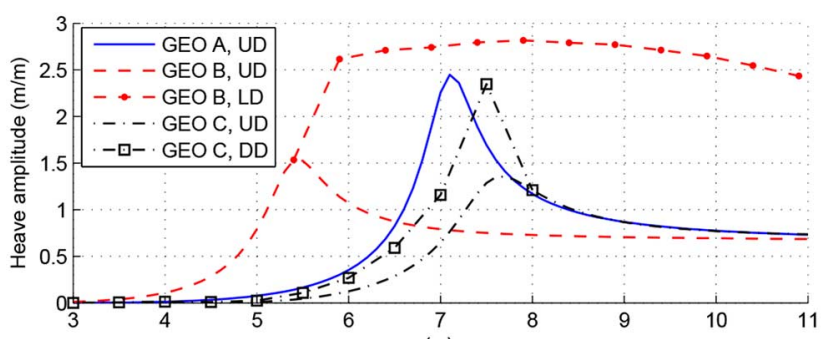

(a)

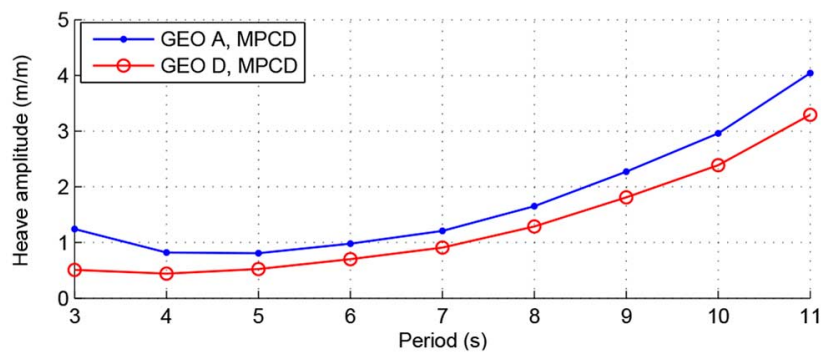

(b)

Fig. 7. RAOs for optimal geometries: (a) GEO A without control, GEO B with latching and without control, and GEO C with declutching and without control; (b) GEO A and GEO D with the MPC-like control algorithm.

\section{CONCLUSion}

In this work, we have studied the implications of geometry optimization in the absence of control and the effects of the incorporation of control design at the device geometry optimization stage. Different optimal geometry results are obtained depending on the type of control strategy employed on the WEC. The effects of constraints on the PTO force or on the amplitude motion of the device have not been taken into account in this study. Such constraints may influence the results, especially in the MPC case, which results in more exaggerated motion of the body and requires higher PTO forces than the latching or declutching strategies.

An optimal geometry obtained with latching control is highly control dependent and it shortens the original natural resonance period of the device, usually designed for the predominant wave period. The optimal geometry obtained with a DD is slightly different from an UD and its resonance period is longer than the uncontrolled case. In these cases, an overall optimal system can be obtained if the latching/declutching control design is considered at the geometry optimization stage. In addition, an optimal controller (like the one considered here) optimizes the wave energy absorption regardless of the geometry of the device. For this case, it has been shown that the same amount of power can be obtained, no matter whether the control design is incorporated at the geometry optimization stage, or not.

In this paper, we have studied the effects of incorporating the control philosophies of latching control, declutching control and an optimal controller for particular sea states and a generic point absorber and PTO system. The effects of these control strategies, and others, on the optimal WEC geometry are likely to differ depending on the type of the WEC and PTO system employed. The control-informed geometric optimization of a real device is specific to the WEC, PTO, sea state(s), and system constraints. For the moment, we simply make the point, 
through the results presented, that the nature of the control system must be considered when designing the WEC itself, if an overall optimal wave energy system is to be achieved.

\section{REFERENCES}

[1] A. F. O. Falcão, "Wave energy utilization: A review of the technologies," Renew. Sustain. Energy Rev., vol. 14, no. 3, pp. 899-918, 2010.

[2] B. Drew, A. R. Plummer, and M. N. Sahinkaya, "A review of wave energy converter technology," in Proc. IMechE Part A Power Energy, vol. 223, no. 8, pp. 887-902, 2009.

[3] K. Budal and J. Falnes, "A resonant point absorber of ocean waves," Nature, vol. 256, pp. 478-479, 1975.

[4] A. Babarit and A. H. Clément, "Optimal latching control of a wave energy device in regular and irregular waves," Appl. Ocean Res., vol. 28, no. 2, pp. 77-91, Apr. 2006.

[5] A. Babarit, M. Guglielmi, and A. H. Clément, "Declutching control of a wave energy converter," Ocean Eng., vol. 36, no. 12-13, pp. 1015-1024, 2009.

[6] A. Babarit and A. Clément, "Shape optimisation of the SEAREV wave energy converter," in Proc. IX World Renewable Energy Congr., Florence, Italy, 2006.

[7] M. Alves, H. Traylor, and A. Sarmento, "Hydrodynamic optimization of a wave energy converter using a heave motion buoy," in Proc. 6th Int. Conf. Wave Tidal Energy, Porto, Portugal, 2007.

[8] A. P. McCabe, G. A. Aggidis, and M. B. Widden, "Optimizing the shape of a surge-and-pitch wave energy collector using a genetic algorithm," Renew. Energy, vol. 35, no. 12, pp. 2767-2775, 2010.

[9] A. P. McCabe, "Constrained optimization of the shape of a wave energy collector by genetic algorithm," Renew. Energy, vol. 51, pp. 274-284, 2013.

[10] A. Kurniawan and T. Moan, "Optimal geometries for wave absorbers oscillating about a fixed axis," J. Ocean. Eng., vol. 38, no. 1, pp. 117-130, 2013.

[11] J.-C. Gilloteaux and J. V. Ringwood, "Control-informed geometric optimisation of wave energy converters," in Proc. 8th IFAC Conf. Control Appl. Mar. Syst., Rostock, Germany, 2010, pp. 399-404.

[12] G. Bacelli and J. V. Ringwood, "Constrained control of arrays of wave energy devices," Int. J. Mar. Energy, vol. 3-4, pp. e53-e69, 2013.

[13] W. E. Cummins, "The impulse response function and ship motions," Schiffstechnik, vol. 47, no. 9, pp. 101-109, 1962.

[14] J. Falnes, Ocean Waves and Oscillating Systems: Linear Interaction including Wave-Energy Extraction. Cambridge, U.K.: Cambridge Univ. Press, 2002.

[15] A. Babarit, G. Duclos, and A. H. Clément, "Comparison of latching strategies for a heaving wave energy device in random sea," Appl. Ocean Res., vol. 26, no. 5, pp. 227-238, Jul. 2004.

[16] G. Nolan, J. V. Ringwood, and W. E. L. S. Butler, "Optimal damping profiles for a heaving buoy wave-energy converter," in Proc. 15th Int. Offshore Polar Eng. Conf., Seoul, Korea, 2005.

[17] J. Hals, T. Bjarte-Larsson, and J. Falnes, "Optimum reactive control and control by latching of a wave-absorbing semisubmerged heaving sphere," in Proc. 21st Int. Conf. Offshore Mech. Arct. Eng., Oslo, Norway, 2002, vol. 8, pp. 415-423.

[18] K. Budal et al., "The Norwegian wave-power buoy project," in Proc. 2nd Int. Symp. Wave Energy Util., Norway, 1982, pp. 323-344.

[19] H. Eidsmoen, "Tight-moored amplitude-limited heaving buoy wave energy converter with phase control," Appl. Ocean Res., vol. 20, no. 3, pp. 157-161, Jun. 1998.

[20] U. A. Korde, "Phase control of floating bodies from an on-board reference," Appl. Ocean Res., vol. 23, no. 5, pp. 251-262, Oct. 2001.

[21] A. F. O. Falcão, "Phase control through load control of oscillating-body wave energy converters with hydraulic PTO system," Ocean Eng., vol. 35, no. 3, pp. 358-366, 2008.
[22] M. F. P. Lopes et al., "Experimental and numerical investigation of nonpredictive phase-control strategies for a point-absorbing wave energy converter," Ocean Eng., vol. 36, no. 5, pp. 386-402, 2009.

[23] Z. Feng and E. C. Kerrigan, "Latching control of wave energy converters using derivative-free optimization," in Proc. IEEE 52nd Annu. Conf. Decis. Control, Florence, Italy, 2013, pp. 7474-7479.

[24] M. Folley and T. J. T. Whittaker, "The effect of sub-optimal control and the spectral wave climate on the performance of wave energy converter arrays," Appl. Ocean Res., vol. 31, no. 4, pp. 260-266, 2009.

[25] J. Hals, J. Falnes, and T. Moan, "A comparison of selected strategies for adaptive control of wave energy converters," J. Offshore Mech. Arct. Eng., vol. 133, no. 3, pp. 031 101-031 113, 2011.

[26] B. Teillant, J.-C. Gilloteaux, and J. Ringwood, "Optimal damping profile for a heaving buoy wave energy converter," in Proc. 8th IFAC Conf. Control Appl. Mar. Syst., Rostock, Germany, 2010, pp. 393-398.

[27] A. H. Clément and A. Babarit, "Discrete control of resonant wave energy devices," Philos. Trans. Roy. Soc. A Math. Phys. Eng. Sci., vol. 370, no. 1959 , pp. $288-314,2012$.

[28] Z. Feng and E. C. Kerrigan, "Declutching control of wave energy converters using derivative-free optimization," in Proc. 19th IFAC World Congr., Cape Town, South Africa, 2013, pp. 7647-7652.

[29] G. Bacelli, J. V. Ringwood, and J.-C. Gilloteaux, "A control system for a self-reacting point absorber wave energy converter subject to constraints," in Proc. 18th IFAC World Congr., Milan, Italy, 2011, pp. 11 387-11 392.

[30] G. Bacelli, P. Balitsky, and J. V. Ringwood, "Coordinated control of arrays of wave energy devices-Benefits over independent control," IEEE Trans. Sustain. Energy, vol. 4, no. 4, pp. 1091-1099, Oct. 2013.

[31] WAMIT, Inc., WAMIT User Manual Versions 6.4, 6.4PC and 6.3S, 6.3S$P C$, Chestnut Hill, MA, USA: WAMIT, Inc., 1998-2006.

[32] J. Labeyrie, "Stationary and transient states of random seas," Mar. Struct., vol. 3, pp. 43-58, 1990.

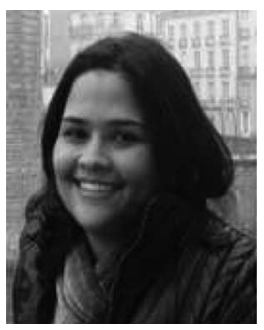

Paula B. Garcia-Rosa (S'13-M'13) received the B.Sc. degree in electronic engineering from the State University of Rio de Janeiro, Rio de Janeiro, Brazil, in 2004, and the M.Sc. degree in electrical engineering and the D.Sc. degree in ocean engineering from the COPPE/Federal University of Rio de Janeiro, Rio de Janeiro, Brazil, in 2008 and 2013, respectively.

Currently, she is a Postdoctoral Researcher with the Centre for Ocean Energy Research, Maynooth University, Maynooth, Ireland. Her research interests include automation and control of industrial processes, renewable energy technologies and modeling, and optimization and control of ocean energy systems.

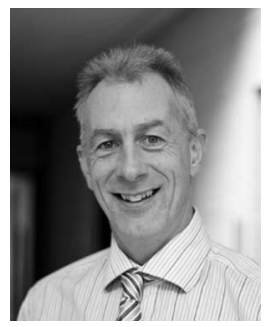

John V. Ringwood (M'87-SM'97) received the Diploma degree in electrical engineering from Dublin Institute of Technology, Dublin, Ireland, in 1981, and the Ph.D. degree in control systems from Strathclyde University, Glascow, U.K., in 1985.

Currently, he is a Professor of Electronic Engineering with the Maynooth University, Maynooth, Ireland. From 2000 to 2006, he was the Head of the Electronic Engineering Department, Maynooth University, developing the department from a greenfield site. His research interests cover time-series modeling, wave energy, control of plasma processes, and biomedical engineering.

Dr. Ringwood is a Chartered Engineer and a Fellow of the Institution of Engineers of Ireland. 